\title{
CORRESPONDENCE
}

\section{Vaccine booster efficiently inhibits entry of SARS-CoV-2 omicron variant}

Wei Zhang ${ }^{1,2,6}$, Linfen Huang ${ }^{1,2,6}$, Gang Ye ${ }^{1,2}$, Qibin Geng ${ }^{1,2}$, Nwando lkeogu ${ }^{3}$, Morgan Harris ${ }^{3}$, Gayathri Dileepan $^{3}$, Kristina Burrack ${ }^{3,4}$, Lanying $\mathrm{Du}^{5}$, Anne Frosch ${ }^{3,4}$ and Fang $\mathrm{Li}\left(\mathbb{D}^{1,2}{ }^{凶}\right.$

(c) The Author(s), under exclusive licence to CSI and USTC 2022

Cellular \& Molecular Immunology (2022) 19:445-446; https://doi.org/10.1038/s41423-022-00837-6

The newly emerged omicron strain of SARS-CoV-2 is spreading fast globally, replacing the previously dominant delta strain in many parts of the world [1]. Compared to the early prototypic strains, the delta strain evades the human immune system more easily, causing many breakthrough infections in vaccinated people [2]. It is imperative to understand in a timely fashion whether the omicron strain escapes immune surveillances. The information will be critical for public health measures. The virus-surface spike protein mediates SARS-CoV-2 entry into host cells and induces most of the host immune responses [3]. The spike protein is also the basis of many COVID-19 vaccines [4]. Particularly, two widely used mRNA vaccines, from Pfizer and Moderna respectively, encode the SARS-CoV-2 spike protein ectodomain. In this study, we investigated how efficiently the omicron spike protein escapes the immune responses from mRNA spike vaccines, using the prototypic strain and delta strain as comparisons.

To investigate the efficiency of the omicron spike in escaping immune responses, we performed pseudovirus assays in the presence of vaccinated sera. Plasma was collected from adult participants at Hennepin Healthcare, Minneapolis, MN at 19-34 days after primary vaccination series and 6-69 days after booster dose. The Institutional Review Board of Hennepin Healthcare Research Institute reviewed and approved the protocols associated with this study. Written informed consent was obtained from all participants. We packaged SARS-CoV-2 pseudoviruses using the omicron spike and used them to enter HEK293T cells stably expressing human ACE2 [5]. As comparisons, we also packaged two other SARS-CoV-2 pseudoviruses using the spikes from a prototypic strain and delta strain, respectively. Sera from two groups of vaccinated people were collected: (i) ten people who had received two doses of the Pfizer mRNA spike vaccine; (ii) ten people who had received three doses of the same vaccine. Serially diluted sera from each vaccinated person were added to the pseudovirus assay and inhibition index $\mathrm{NT}_{50}$ was calculated for sera from each person. The result showed that omicron pseudoviruses were inhibited inefficiently by doubly vaccinated sera: $\sim 4$ times less efficiently compared to the delta strain and $\sim 8$ times less efficiently compared to the prototypic strain (Fig. 1). Moreover, omicron pseudoviruses were inhibited $\sim 10$ times more efficiently by triply vaccinated sera than by doubly vaccinated sera. Thus, whereas the omicron spike demonstrates enhanced immune escape, booster vaccinations are useful in protection against omicron spike-mediated viral entry.

Our study provides timely characterization of the immune escape of the omicron spike from vaccinated sera. The enhanced immune escape of the omicron spike protein is consistent with reports that compared to the previous strains, the omicron strain spreads more easily in human populations including vaccinated people [1]. Moreover, the booster vaccines induce more effective immune responses against the omicron spike than two-dose vaccination, supporting the Center for Disease Control (CDC)'s recommendation for booster vaccination [6].

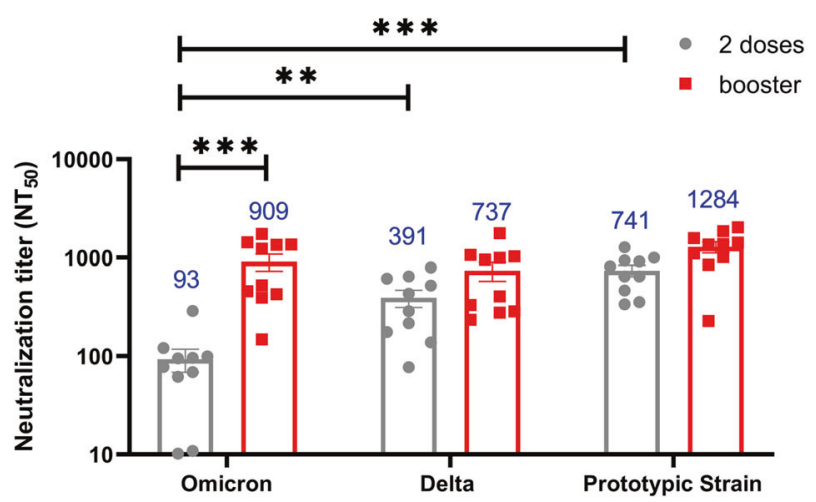

Fig. 1 Inhibition of omicron pseudoviruses by vaccinated sera. Sera were collected from two groups of vaccinated people. In one of the groups, ten people received two doses of the Pfizer mRNA spike vaccine. In the other group, ten people received three doses of the same vaccine. SARS-CoV-2 pseudoviruses were used to infect HEK293T cells stably expressing human ACE2 in the presence of serially diluted sera. $\mathrm{NT}_{50}$ values (neutralization titer that inhibits pseudovirus entry by $50 \%$ ) were calculated for sera from each person. All the measurements were carried out in triplicates. The values are presented as mean plus standard error of the mean (SEM). A Student's two-tailed $t$ test was performed to analyze the statistical differences among the noted groups. ${ }^{*}{ }^{*} P<0.001 ;{ }^{*} P<0.01$. The experiments were repeated three times with similar results.

\footnotetext{
${ }^{1}$ Department of Veterinary and Biomedical Sciences, University of Minnesota, Saint Paul, MN, USA. ${ }^{2}$ Center for Coronavirus Research, University of Minnesota, Saint Paul, MN, USA.

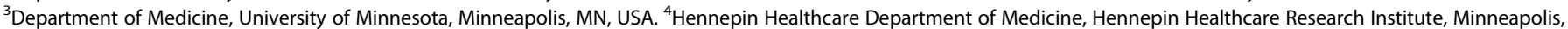
MN, USA. ${ }^{5}$ Institute for Biomedical Sciences, Georgia State University, Atlanta, GA, USA. ${ }^{6}$ These authors contributed equally: Wei Zhang, Linfen Huang.

email: anne.frosch@hcmed.org; lifang@umn.edu
}

Received: 4 January 2022 Accepted: 10 January 2022

Published online: 24 January 2022 


\section{REFERENCES}

1. SARS-CoV-2 B.1.1.529 (Omicron) Variant - United States, December 1-8, 2021. MMWR. Morbidity and mortality weekly report. 2021;70:1731-4. https://doi.org/ 10.15585/mmwr.mm7050e1.

2. Lopez Bernal J, Andrews N, Gower C, Gallagher E, Simmons R, Thelwall S, et al. Effectiveness of Covid-19 Vaccines against the B.1.617.2 (Delta) Variant. N. Engl J Med. 2021;385:585-94. https://doi.org/10.1056/NEJMoa2108891.

3. Li F. Structure, function, and evolution of coronavirus spike proteins. Annu Rev Virol. 2016;3:237-61. https://doi.org/10.1146/annurev-virology-110615-042301.

4. Krammer F. SARS-CoV-2 vaccines in development. Nature. 2020;586:516-27. https://doi.org/10.1038/s41586-020-2798-3.

5. Geng Q, Tai W, Baxter VK, Shi J, Wan Y, Zhang X, et al. Novel virus-like nanoparticle vaccine effectively protects animal model from SARS-CoV-2 infection. PLoS Pathog. 2021;17:e1009897. https://doi.org/10.1371/journal.ppat.1009897.

6. Kalter, L. CDC: Boosters Key in Protecting Against Omicron, https://www.webmd. com/vaccines/covid-19-vaccine/news/20211210/boosters-key-for-omicron (2021).

\section{AUTHOR CONTRIBUTIONS}

Investigation: $\mathrm{WZ}, \mathrm{LH}, \mathrm{GY}$, and $\mathrm{QG}$ performed pseudovirus entry assay; $\mathrm{MH}, \mathrm{NI}$, and GD collected and processed all the specimens, and managed database. Project administration: FL, AF, LD, and KB. Manuscript writing: FL. Manuscript review and editing: $A F$ and LD. Funding acquisition: $F L, A F$, and LD. Resources: $F L$ and AF.

\section{FUNDING}

This study was supported by grants R01Al089728 and R01Al110700 (to F.L.), R01Al139092 (to F.L. and L.D.) and 5K08Al141761 (to A.F.). The funders had no role in the design or conduct of the study; collection, management, analysis, and interpretation of the data; preparation, review, or approval of the manuscript; or the decision to submit the manuscript for publication.

\section{COMPETING INTERESTS}

The authors declare no competing interests.

\section{ADDITIONAL INFORMATION}

Supplementary information The online version contains supplementary material available at https://doi.org/10.1038/s41423-022-00837-6.

Correspondence and requests for materials should be addressed to Anne Frosch or Fang Li.

Reprints and permission information is available at http://www.nature.com/ reprints 